\title{
基于液态金属的可印刷式热电发生器及其 性能评估
}

李海燕 ${ }^{(1)}$, 周远 ${ }^{(1)}$, 刘静 ${ }^{(1)}$ (2)

(1) 中国科学院理化技术研究所低温工程学重点实验室, 北京 100190;

(2) 清华大学医学院生物医学工程系, 北京 100084

*E-mail: jliu@mail.ipc.ac.cn

收稿日期: 2013-12-10; 接受日期: 2013-12-17

国家重点基础研究发展计划(“973”计划)(批准号: 2010CB227303)资助项目

摘要液态金属印刷电子学作为正在兴起的电子直写技术, 在各类柔性电子电路、光电传感 器乃至能量捕获器的快速制造上已显示出较大的发展潜力. 提出基于液态金属电子墨水的可 印刷式热电发生器概念, 并由此研制了对应的集成器件. 特别以镓-康铜热电偶及镓-镓铟合金 热电偶为代表，系统评估了采用液态金属直写方法制作的液-固、液-液两类组合模式的热电偶 及热电堆的热电性能, 并篮选出合适的配对材料; 继而将 20 对镓-康铜热电偶集成为热电发生 器, 对其输出电压予以放大后得到 $1.59 \mathrm{~V}$ 的负载电压和 $70.44 \mu \mathrm{W}$ 的功率, 可成功驱动 LED 灯. 在此基础上, 进一步地将镓膜层的厚度从 $10 \mu \mathrm{m}$ 提升至 $800 \mu \mathrm{m}$, 由此将负载电压和功率增至 $1.70 \mathrm{~V}$ 和 $742.9 \mu \mathrm{W}$, 幅度分别达到 $6.92 \%$ 和 $955 \%$. 以上一系列基础和应用实验证明了液态金 属直写式热电发生器用于热量捕获的实用价值.

关键词

热电发生器 热量捕获 印刷电子学 液态金属 直写技术

\section{1 引言}

近年来, 随着化石燃料的大量消耗和日益枯竭, 开发利用清洁能源以避免生态环境进一步恶化成为 大势所趋. 热电材料和器件的开发利用, 通过无污染 的热电转换效应, 将来自地热、太阳光、汽车尾气、 工业废热等的热能转换成生产生活用电, 逐渐被人 们寄予厚望 ${ }^{[1 ~ 4]}$. 与其他热机相比, 热电发生器体积 小得多, 尽管其目前的转化效率偏低, 仍不失为一种 很有前景的能源技术. 根据可直接转换成电能的潜 在热量多少, 热电发生器的应用一般分为微观和宏
观两类. 前者包括驱动自适应微系统(如微芯片 ${ }^{[5]}$ 、无 线传感器网络 ${ }^{[6,7]}$ )和可穿戴或可植入式电子设备(如 助听器、腕表 ${ }^{[8,9]}$ 、起搏器 ${ }^{[10]}$ ); 宏观余热应用则包括 家用 ${ }^{[11]}$ 、汽车 ${ }^{[12]}$ 、工业及固体废弃物 ${ }^{[4]}$ 等.

传统的热电装置一般是由热沉板、吸热板和热电 堆组成的“三明治”结构. 受硬板所限, 主要用于平面 热源. 为了能与任意结构热源均达到良好的热接触, 开发可适应各种表面的热电模块成为必然趋势. 柔 性热电发生器因此应运而生 ${ }^{[13,14]}$. 此外, 通过薄膜沉 积技术, 人们还探索采用纳米材料以提高热电薄膜 效率. 目前形式各异且各具特色的薄膜制备方法正

引用格式: 李海燕, 周远, 刘静. 基于液态金属的可印刷式热电发生器及其性能评估. 中国科学: 技术科学, 2014, 44: 407-416, doi: 10.1360/092013-1239 Li H Y, Zhou Y, Liu J. Liquid metal based printable thermoelectronic generator and its performance evaluation (in Chinese). Sci Sin Tech, 2014, 44: 407-416, doi: 10.1360/092013-1239 
为热电科学的发展创造条件. 常用的热电薄膜沉积 技术往往比较复杂、耗时长、成本高. 与之相比, 近 年来出现的直写技术产量高、耗材少且对基底材料要 求低 ${ }^{[15,16]}$, 从长远看具有明显的发展优势.

与常规的热电材料相比，室温液态金属具有电 导率高、材料兼容性好、室温下呈液态, 可直接印刷 在各种柔性基底上制成任意形状和厚度的器件等特 点, 大大降低了制作难度及成本, 令其成为热能捕获 领域中一种较有应用前景的材料. 正是基于这一认 识，本文首次探索了采用直写方法制作液态金属热 电发生器的可行性, 对由不同配对金属构成的热电 偶的热电性能进行了测试, 并制作了由 20 对热电偶 串联而成的液态金属热电发生器原型机, 证明了可 印刷式液态金属热电发生器的实用价值.

\section{2 热电材料性能评价指标}

热电材料又称温差电材料, 是一种利用固体内 部载流子的运动实现热能和电能直接相互转换的功 能材料 ${ }^{[17]}$, 其性能可用热电优值系数来评估 ${ }^{[18]}$ :

$$
Z=\frac{S^{2}}{\kappa \cdot \lambda},
$$

其中 $Z$ 为热电优值; $S$ 为 Seebeck 系数或热电势率; $\kappa$ 为电阻率; $\lambda$ 为热导率. 由于不同环境温度下材料的 $Z$ 值不同, 人们习惯上常用热电系数与温度之积一无 量纲热电优值 $Z T$ 的大小来描述在一定温度 $T$ 时热电 材料性能的好坏 ${ }^{[19]}$, 即

$$
Z T=\frac{S^{2}}{\kappa \cdot \lambda} T,
$$

其中 $T$ 是绝对温度. (2)式中 $T$ 亦可换成 $\bar{T}(\bar{T}=$ $\left.\left(T_{H}+T_{L}\right) / 2\right)$, 用以表示一定温度范围内热电材料性 能的好坏, $T_{H}, T_{L}$ 分别为热端、冷端温度.

从(2)式可发现, 热电材料的 Seebeck 系数越高, 电阻率越低, 热导率越低, $Z T$ 值越高. 提高热电材料 的优值系数的途径主要是通过提高载流子浓度和载 流子迁移率, 以提高热电半导体材料的电导率. 但实
验证明, 对于许多热电材料, 电导率提高到一定值后, 热电势率却随着电导率的进一步提高而大幅下降 ${ }^{[20]}$. 另外, 电导率的增加还会引起热导率的提高, 从而引 起材料两端温差下降乃至降低热电转换效率. 目前 业界研究热点集中在通过降低声子热导率来减小材 料的热导率. 其中主要是利用分子束外延、CVD、激 光熔融、高压粒子注入、粒子电化学沉积等工艺，制 备薄膜和低维量子结构, 通过提高其声子散射, 从而 有效降低热导率. 常用热电材料的 $Z T$ 值一般为 1, 而近来报道的新型热电材料的 $Z T$ 值逐渐提高, 已接 近 $2.5^{[21 ~ 23]}$

对于热电薄膜发电机, 热电优值 $Z$ 的简化版更常 用, 称为功率因子 $w\left(\mathrm{~W} / \mathrm{K}^{2} \mathrm{~m}\right)$, 即 ${ }^{[24]}$

$$
w=\frac{S^{2}}{\kappa} \text {. }
$$

根据上述分析, 本文试验研究了所提出的新型可 印刷式热电器件的一系列物理特性.

\section{3 直写式液-固热电偶热量捕获器性能}

\section{1 实验材料}

根据文献[25]的研究结论, 康铜与液态镓构成的 热电偶具有相对较大的热电势率 $(47.64 \mu \mathrm{V} / \mathrm{K})$, 故选 择液态镓与固态康铜丝构成的热电偶作为研究对象. 考虑到要输出尽可能高的电功率, 热电偶的总电阻 应尽量低. 根据电阻公式 $R=\kappa \cdot L / A$, 在电阻率 $\kappa$ 一 定的前提下, 热电材料的长度 $L$ 应尽量小, 截面积 $A$ 则应尽量大. 故而从上海拓群工业陶瓷厂专门购置 了直径 $1.5 \mathrm{~mm}$ 的康铜丝, 选取长度 $100 \mathrm{~mm}$, 采用四 端子法测得其实际电阻并换算得到电阻率为 $\kappa=5.35 \times$ $10^{-7} \Omega \mathrm{m}$, 与理论值 $\kappa=4.8 \times 10^{-7} \Omega \mathrm{m}$ 相近. 液态金属 镓从厂家购得，纯度为 $99.99 \%$. 基底材料选用与液态 金属相容性极佳的柔性硅胶板，尺寸 $15 \mathrm{~mm} \times 12 \mathrm{~mm} \times$ $3 \mathrm{~mm}$ ，用无水乙醇清洁后备用.

镓和康铜的物理性质及实验参数如表 1 所示. 其 中康铜电阻率为实测数据, 其余物性数据来自文献.

\section{表 1 被试材料的物理性质及参数 [26 29]}

\begin{tabular}{cccccc}
\hline 材料 & 电阻率 $\kappa(\Omega \mathrm{m})$ & 热导率 $\lambda(\mathrm{W} / \mathrm{K} \mathrm{m})$ & 热电势率 $S(\mu \mathrm{V} / \mathrm{K})$ & 长度 $L(\mathrm{~m})$ & 截面积 $A\left(\mathrm{~m}^{2}\right)$ \\
\hline 镓 & $2.72 \times 10^{-7}$ & 29.4 & -0.2 & 0.1 & $1 \times 10^{-7}$ \\
康铜 & $5.08 \times 10^{-7}$ & 21.2 & -35 & 0.1 & $1.77 \times 10^{-6}$ \\
\hline
\end{tabular}




\section{2 实验平台和方法}

实验平台如图 1 所示, 实验方法如下.

1) 采用 $10 \mathrm{~mL}$ 浓度为 $30 \%$ 的 $\mathrm{NaOH}$ 溶液对镓进 行预处理并在磁力搅拌器上搅拌 $10 \mathrm{~min}$ 后, 获得含 有氧含量为 $0.026 \mathrm{wt} . \%$ 的镓.

2) 借助 PVC (聚氯乙烯)板制成的掩膜在硅胶板 上用笔直接画出宽 $10 \mathrm{~mm}$ 、长 $100 \mathrm{~mm}$ 的镓薄膜.

3) 在镓膜两端各放置一段 $50 \mathrm{~mm}$ 长的康铜丝, 使 两段康铜丝的一端分别与镓膜端部相接, 另一端空置.

4) 用透明 705 硅橡胶对镓膜部分进行封装.

5) 将上述硅胶板用硅橡胶固定在相隔一定距离 的两铝块上, 保证镓和康铜丝两接点分别位于其上, 两康铜丝空置端则固定于一独立铝块上以确保均温. 铝块表面采用聚酰亚胺覆盖以防止短路。

6) 通过调压器调节铝块中加热棒的温度, 使其 中镓-康铜一接点温度保持 $30^{\circ} \mathrm{C}$, 另一接点温度持续 上升至 $230^{\circ} \mathrm{C}$, 于是在镓膜两侧建立起温差 $\Delta T$. 两铝 块中间用多层聚四氟乙烯薄膜隔热，镓膜中部用加 热贴覆盖, 保持其温度始终在 $50^{\circ} \mathrm{C}$ 左右, 以保证镓 始终为单相液体.

7) 用精度为 $\pm 0.5^{\circ} \mathrm{C}$ 的 $\mathrm{T}$ 型热电偶实时监测接点 温度和镓膜线路中温度, 测压线路接入两康铜丝空 置端以测量开路电压. 接入负载后分别采集热电偶 的输出电压和负载电压. 用安捷伦 $34970 \mathrm{~A}$ 数据采集 装置采集并记录温度和电压数据. 根据测得的负载 电压和负载电阻值可计算不同温差下的输出功率.

\section{3 镓-康铜热电偶热电性能}

根据塞贝克效应，热电偶热电势与温差存在如 下关系:

$$
E=S_{\mathrm{AB}} \Delta T,
$$

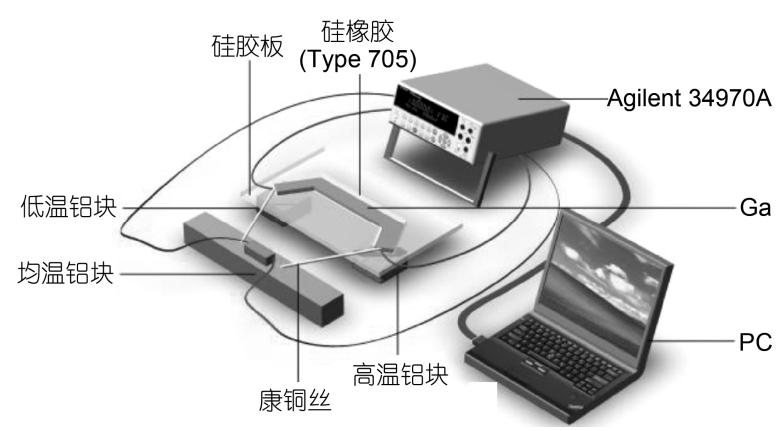

图 1 热电捕获实验平台
其中 $S_{\mathrm{AB}}$ 为 $\mathrm{A}, \mathrm{B}$ 两种材料对应的热电势率之差.

图 2(a)为热电势(即开路电压)随温差的变化关系. 从中可以看出液态镓薄膜和康铜丝构成的热电偶输 出的电压与温差呈现出非常好的线性关系.

镓-康铜热电偶的内阻可由下式估算:

$$
R_{\mathrm{o}}=R_{\mathrm{Ga}}+R_{\mathrm{con}}=\frac{\kappa_{\mathrm{Ga}} \cdot L_{\mathrm{Ga}}}{A_{\mathrm{Ga}}}+\frac{\kappa_{\mathrm{Con}} \cdot L_{\mathrm{Con}}}{A_{\mathrm{Con}}},
$$

其中 $R_{\mathrm{o}}, R_{\mathrm{Ga}}$ 和 $R_{\mathrm{con}}$ 分别为镓-康铜热电偶的内阻以及 镓和康铜的电阻. 由(5)式可计算得热电偶内阻为 $0.18 \Omega$. 根据最大功率传输定理：一个含源二端网络 对负载电阻供电, 当负载电阻与该含源二端网络的 等效内阻相等时, 负载电阻上获得最大功率. 于是为 获得尽可能大的输出功率，选用与热电偶内阻相对 接近的阻值为 $0.1 \Omega$ 的负载电阻接入构成闭合电路.

图 2(b)为不同温度梯度下的负载电压及输出功 率曲线. 可见，在温度梯度为 $200^{\circ} \mathrm{C}$ 时得到了最大负 载电压 $4.23 \mathrm{mV}$ 和最大输出功率 $179 \mu \mathrm{W}$, 而且多次 实验显示出良好的可重复性. 功率曲线呈现出抛物线
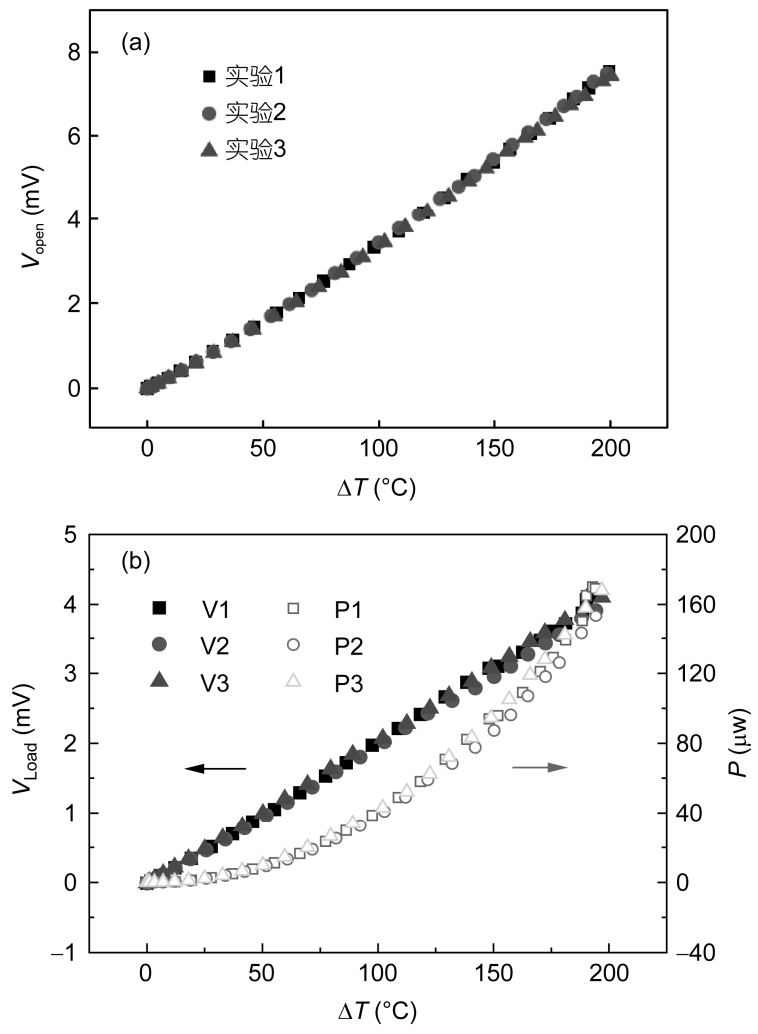

图 2 镓-康铜热电偶热电性能

(a) 开路电压随温差变化关系; (b) 负载电压和输出功率随温差变化 关系 
特性, 虽然只有微瓦级, 但对手表、助听器等小型电子 设备已足可应用 ${ }^{[30]}$ (如手表只需 $1 \sim 2 \mu \mathrm{W}$ ), 这就为镓康铜热电偶在小温差下的可穿戴应用提供了可能.

从以上研究亦可知, 倘若提高温度梯度, 应该可 以获得更大的热电势和输出功率, 但鉴于本实验所 采用的基底材料在高温下易于分解, 若要获得更大 的热电势和功率, 需要进一步寻求耐高温且和液态 金属相容性好的基底材料.

\section{4 性能指标}

性能指标是评价热电材料和热电偶性能优劣的 主要判据. 根据(1) (3)式, 可计算得到镓、康铜以及 二者组成的热电偶的热电性能指标, 包括热电优值 $Z$ 、无量纲热电优值 $Z T$ 和功率因子 $w$, 具体结果见 表 2 , 这里用镓的相关参数近似作为含微量氧化物的 镓的参数.

从表 2 中发现, $303 \mathrm{~K}$ 时镓和康铜的 $Z T$ 值与目前 常用的 $\mathrm{Bi}_{2} \mathrm{Te}_{3}$ (室温下 $Z T$ 值可达到 1 左右 ${ }^{[9]}$ )相比较 低, 这可以归结于二者相对更高的热导率, 而镓同时 受限于其较低的热电势率, 所以其热电性能似乎并 不占优势. 但其与现有热电材料相比, 具有独特的可 印刷特性, 可通过参数调整来改变热电极特定的印 刷长度、宽度和厚度, 实现热电偶的快速定制. 在某 些特殊场合, 基于液态金属的热电发生器较具利用 价值. 可通过掺杂等方式制备热电优值高的印刷材 料, 从而在保持液态金属可印刷性的基础上, 进一步 提高热电发生器的能量转换效率.

\section{4 直写式液-液热电偶热量捕获器性能}

如下采用镓和镓铟合金两种液态金属构成热电 偶, 研究其热电效率, 相应结果也可为其他液态材料 构成的直写式热电发生器提供参考.

\section{1 实验材料与方法}

本实验选用液态金属镓与镓铟合金 $\left(\mathrm{GaIn}_{24.5}\right)$ 构

\section{表 2 性能指标 (303 K)}

\begin{tabular}{lccc}
\hline \multicolumn{1}{c}{ 材料 } & $\begin{array}{c}\text { 热电优值 } Z \\
\left(\mathrm{~K}^{-1}\right)\end{array}$ & $\begin{array}{c}\text { 无量纲热电优值 } \\
Z T\end{array}$ & $\begin{array}{c}\text { 功率因子 } w \\
\left(\mathrm{~W} / \mathrm{K}^{2} \mathrm{~m}\right)\end{array}$ \\
\hline 镓 & $5.00 \times 10^{-9}$ & $1.52 \times 10^{-6}$ & $1.47 \times 10^{-7}$ \\
康铜 & $1.14 \times 10^{-4}$ & $4.41 \times 10^{-2}$ & $4.28 \times 10^{-3}$ \\
镓-康铜 & $3.82 \times 10^{-5}$ & $1.16 \times 10^{-2}$ & $1.83 \times 10^{-3}$ \\
\hline
\end{tabular}

成热电偶. 液态金属镓和铟均从厂家购得, 纯度均为 99.99\%. 基底材料仍采用柔性硅胶板, 尺寸 $15 \mathrm{~mm} \times$ $18 \mathrm{~mm} \times 3 \mathrm{~mm}$, 用无水乙醇清洁后备用.

镓和镓铟合金墨水的制备方法如文献[25]所示. $\mathrm{GaIn}_{24.5}$ 的物理性质如表 3 所示. 其中电阻率为实测 数据, 热导率数据来自文献, 热电势率由实测得到的 镓-镓铟合金热电偶的热电势率与镓的热电势率之差 推得.

实验平台与图 1 类似, 只是将康铜丝替换为镓铟 合金. 这里, 镓铟合金和镓均借助 PVC (聚氯乙烯)板 制成的掩膜在硅胶板上用笔直接写出, 镓膜宽度和 长度仍为 10 和 $100 \mathrm{~mm}$, 镓铟合金分两部分写出, 宽 度为 $10 \mathrm{~mm}$, 每部分长度为 $50 \mathrm{~mm}$. 但需注意的是在 硅胶板固定到铝块之前, 镓膜和镓铟合金膜并不相 接, 以防止在硅胶板移动过程中, 两种金属流动混合. 待硅胶板固定后, 将镓和镓铟合金相邻部分予以连 接, 并将写有镓铟合金空置端的硅胶基底固定于铝 块上予以均温. 最后, 用 705 硅橡胶对镓膜和镓铟合 金膜进行封装.

\section{2 测试结果}

图 3(a)为无负载情形下镓-镓铟合金热电偶开路 电压随温差的变化关系. 已知 $\mathrm{Ga}$ 和 $\mathrm{GaIn}_{24.5}$ 的热电势 率很小, 从图 3 中亦可看出类似的结果, 3 组实验中 热电势率 $S_{\text {Ga-Galn } 245}$ 最大值仅为 $0.076 \mu \mathrm{V} / \mathrm{K}$. 不过总体 而言, 镓-镓铟合金热电偶输出电压与温差呈现出较 好的线性关系. 只是在温差大于 $140^{\circ} \mathrm{C}$ 之后, 3 组实 验均表现出一定程度的输出电压衰减, 推测这是由 于高温时加热铝块上的黏结硅胶融化, 导致基底材 料部分脱离铝块造成输入温度骤降造成. 与镓-康铜 热电偶相比, 镓-镓铟合金热电偶在整个测试过程中 呈现出更大的不稳定性, 推测这是由于其输出电压 过小, 与电压扰动的量级接近, 从而使得任何轻微的 扰动都能明显反映出来.

应该指出的是，由于镓铟合金电阻率高于康铜， 因此, 与镓-康铜热电偶内阻相比, 镓-镓铟合金热电 偶的内阻应更大一些. 不过, 为对比分析起见, 针对

\section{表 $3 \mathrm{GaIn}_{24.5}$ 的物理性质及参数 ${ }^{[27]}$}

\begin{tabular}{cccccc}
\hline 材料 & $\begin{array}{c}\text { 电阻率 } \kappa \\
(\Omega \mathrm{m})\end{array}$ & $\begin{array}{c}\text { 热导率 } \lambda \\
(\mathrm{W} / \mathrm{K} \mathrm{m})\end{array}$ & $\begin{array}{c}\text { 热电势率 } S(\mu \mathrm{V} / \mathrm{K}) \\
(\mathrm{m}) \text { 度 } L\end{array}$ & $\begin{array}{c}\text { 截面积 } A \\
\left(\mathrm{~m}^{2}\right)\end{array}$ \\
\hline $\mathrm{GaIn}_{24.5}$ & $2.98 \times 10^{-7}$ & 24.3 & -0.31 & 0.1 & $1 \times 10^{-7}$ \\
\hline
\end{tabular}



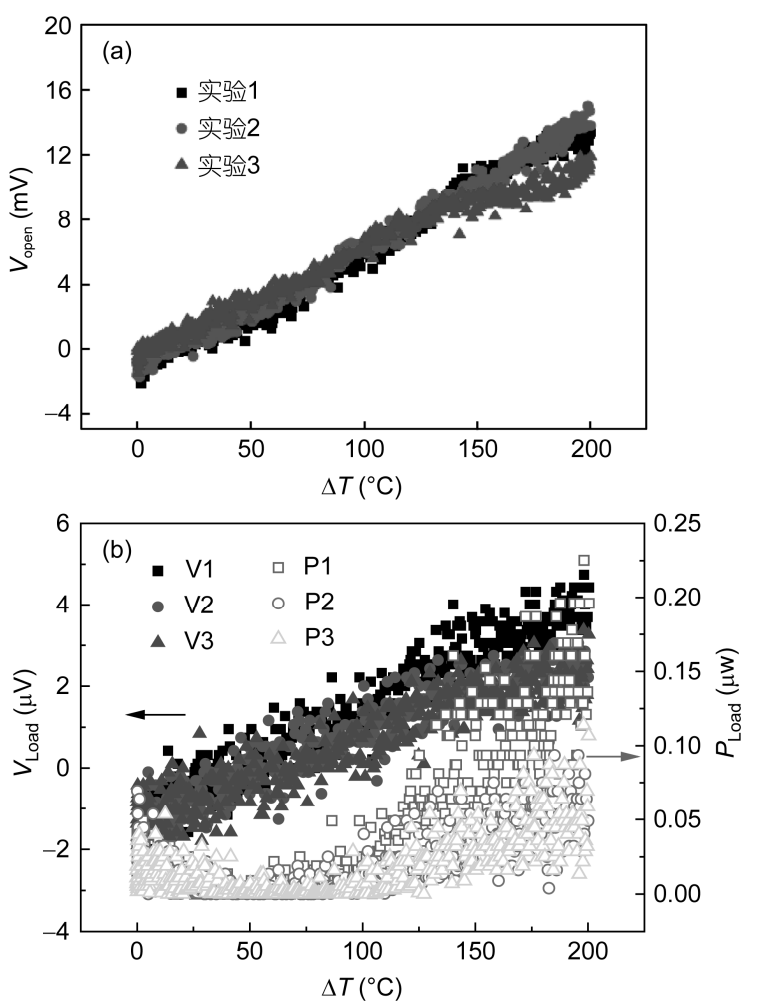

图 3 镓-镓铟合金热电偶热电性能

(a) 开路电压情况; (b) 有负载情形

带负载情形, 此处电路仍然选用 $0.1 \Omega$ 的电阻加以测 试. 图 3(b)为不同温度梯度下镓-镓铟合金热电偶的 负载电压、输出功率曲线. 从中可以看出, 在温度梯 度为 $200^{\circ} \mathrm{C}$ 时得到的最大负载电压和最大输出功率 分别仅为 $4.43 \mu \mathrm{V}$ 和 $0.225 \mathrm{nW}$, 在温差大于 $140^{\circ} \mathrm{C}$ 之 后, 3 组实验呈现出输出电压衰减状况, 原因同前. 而且随着实验次数的递增, 负载电压和功率也呈现 出一定程度的衰减. 实验中观察到硅胶基底在近 $200^{\circ} \mathrm{C}$ 的高温下已出现分解, 加热铝块上方的封装用 705 硅橡胶可见清晰气泡, 可判断基底的分解一定程 度上破坏了封装结构, 可能有部分空气进入封装结 构内部，与高温共同作用致使镓和镓铟合金氧化, 由 于二者的热电性能同时发生变化, 且热电性能绝对 值很小, 所以镓-镓铟合金热电偶的性能衰减明显. 虽然镓-康铜热电偶实验中亦出现硅胶基底分解状况, 但由于镓-康铜热电偶的热电势率较大, 所以从趋势 图中并未观察到基底的影响. 在图 3(b)中可同时发现, 虽然第 2 次实验中出现性能明显下降的情况, 但第 3 次实验结果却与第 2 次实验结果类似. 推测是由于硅
胶材料分解结束, 封装结构亦已趋于稳定, 故而性能 基本保持与第 2 次相近. 可见, 基底材料的选择对两 种液态金属构成的热电偶的热电性能有着至关重要 的影响, 当然, 如果工作温度在 $100^{\circ} \mathrm{C}$ 以下, 包括硅 胶在内的大部分材料都可采用. 另外, 在高温下虽然 功率曲线呈现出预想中的抛物线特性, 但由于最大 输出功率甚至不足纳瓦量级, 单个镓-镓铟合金热电 偶无法满足实际使用需求. 鉴于升高温度对提升热 电势和输出功率的效果有限, 而且更高温度会引发 更为严重的氧化效应，同时对基底材料的要求更高， 所以不建议采取此方式. 将热电偶串联形成热电堆 是达到更大热电势和输出功率的有效方法, 不过需 要数千对镓-镓铟合金热电偶串联才可以获得可供利 用的能量.

\section{3 性能指标}

镓、镓铟合金以及二者组成的热电偶的热电性能 指标, 包括热电优值 $Z$ 、无量纲热电优值 $Z T$ 和功率 因子 $w$ ，见表 4 . 从中发现, $303 \mathrm{~K}$ 时镓铟合金与镓的 $Z T$ 值在同一量级, 明显低于常用热电材料, 二者同 样拥有热导率高和热电势率低的缺陷, 直接导致所 构成的热电偶性能低下. 可见, 材料性能的改进对两 种液态金属组合成的热电偶的实用化具有更为重要 的意义.

\section{5 实用化液态金属直写式热电发生器的研 制及应用试验}

单个热电偶的温差电动势往往较低, 实际使用 中需要采用多个相同热电偶构成热电堆(亦称“温差 电堆”), 通常这些热电偶在电路上是串联的, 而在传 热方面则是并列的, 即在结构布置中使其冷端在热 电堆的一侧, 而热端在另一侧, 这两侧分别称为热电 堆的“冷端”和“热端”. 在相同温差下, 热电堆温差电 动势为所有串联热电偶温差电动势的叠加, 由此可

\section{表 4 性能指标(303 K)}

\begin{tabular}{lccc}
\hline \multicolumn{1}{c}{ 材料 } & $\begin{array}{c}\text { 热电优值 } Z \\
\left(\mathrm{~K}^{-1}\right)\end{array}$ & $\begin{array}{c}\text { 无量纲热电优 } \\
\text { 值 } Z T\end{array}$ & $\begin{array}{c}\text { 功率因子 } w \\
\left(\mathrm{~W} / \mathrm{K}^{2} \mathrm{~m}\right)\end{array}$ \\
\hline 镓 & $5.00 \times 10^{-9}$ & $1.52 \times 10^{-6}$ & $1.47 \times 10^{-7}$ \\
镓铟合金 & $1.33 \times 10^{-8}$ & $4.02 \times 10^{-6}$ & $3.22 \times 10^{-7}$ \\
镓-镓铟热电偶 & $1.90 \times 10^{-10}$ & $5.75 \times 10^{-8}$ & $1.01 \times 10^{-8}$ \\
\hline
\end{tabular}


提供更高的输出电压和功率. 为进一步揭示液态金 属在热量捕获领域的应用价值, 如下对以直写方式制 作的液态金属热电发生器的热量捕获性能进行研究.

\section{1 液态金属直写式热电发生器性能}

之前已证明镓-康铜热电偶的热电性能远高于镓 -镓铟合金热电偶的热电性能, 为得到较大电压和功 率, 这里选用镓-康铜热电偶制成热电发生器.

可印刷热电发生器的制作步骤如下：1）首先用 $0.15 \mathrm{~mm}$ 厚的 PVC 板根据所需形状制作掩膜, 形成 20 个槽道;2) 以 $1 \mathrm{~mm}$ 厚的硅胶板作为基底, 在其上 覆盖掩膜, 用笔刷将液态镓根据槽道形状涂覆成薄 膜；3）取下掩膜, 将康铜丝搭接在每两个镓薄膜之 间, 保证康铜丝两端与镓薄膜两端相接；4）最后，使 用透明 705 硅橡胶封装镓部分, 完成装置制作.

图 4(a)为一个由 20 对镓-康铜热电偶组成的热电 发生器的原型, 镓热电极尺寸为 $100 \mathrm{~mm} \times 10 \mathrm{~mm} \times$ $10 \mu \mathrm{m}$, 康铜热电极尺寸 $100 \mathrm{~mm} \times \Phi 1.5 \mathrm{~mm}$, 热电极 间距 $5 \mathrm{~mm}$.

实验中, 两相隔 $450 \mathrm{~mm}$ 的铝块(尺寸 $300 \mathrm{~mm} \times$ $20 \mathrm{~mm} \times 10 \mathrm{~mm}$ ) 分别为热电发生器提供冷却和加热表
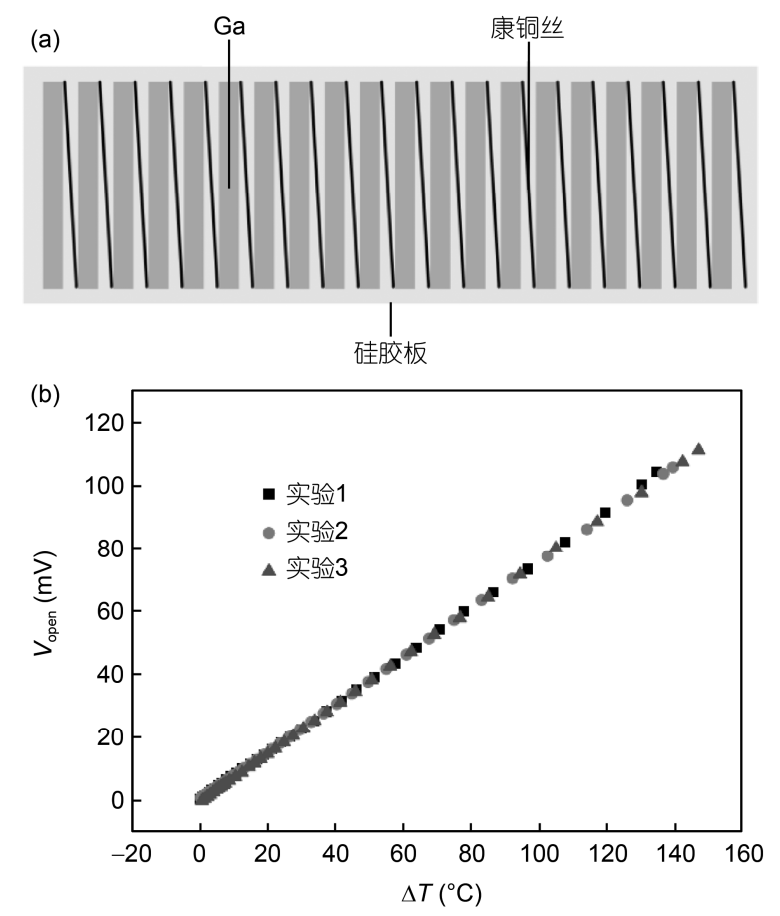

图 4 镓-康铜热电发生器及性能

(a) 原型机; (b) 热电势-温差关系
面, 并起支撑固定作用. 热电发生器的硅胶基底通过 硅橡胶黏接在两铝块上方, 并保证热电偶热端和冷 端分别位于两铝块正上方. 铝块下方由平板加热器 提供热量, 热界面材料用于铝块和平板加热器的接 触表面以减少接触热阻. 使用 $\mathrm{T}$ 型热电偶监测温度, 安捷伦 $34970 \mathrm{~A}$ 采集温度和电压数据.

图 4(b)所示为本文开发的镓-康铜热电发生器原 型机的热电势-温差关系曲线.

根据热电堆输出电压的基本公式, 热电堆的输 出电动势为各串联热电偶输出热电势的叠加, 即

$$
E_{n}=n S_{\mathrm{AB}} \Delta T,
$$

其中 $n$ 为串联的热电偶个数.

对比图 2 和 4 的结果看到, 当温差为 $140^{\circ} \mathrm{C}$ 时, 20 个同样尺寸的热电偶串联可得到输出电压约为 $105 \mathrm{mV}$, 近似等于同样温差下单个热电偶输出电压 (约 $5.05 \mathrm{mV}$ )之和, 显示了热电偶形状尺寸的可控性.

\section{2 液态金属直写式热电发生器驱动 LED 灯实验}

如下采用一个 LED 灯作为负载, 以证明以上研 制的热电发生器的实用价值. 由于 LED 灯的工作电 压是 $2 \mathrm{~V}$, 约为现有输出电压的 20 倍, 所以由该热电 发生器直接驱动 LED 灯并不可行. 为满足所需功率 要求, 我们采用一个商用超低输入电压的升压型 $\mathrm{DC} / \mathrm{DC}$ 转换器(LTC 3108, 凌力尔特), 来将 $\mathrm{mV}$ 级输 入电压放大至几伏的量级, 从而驱动 LED 灯. 该转 换器的最小输入电压为 $20 \mathrm{mV}$, 并提供了四种可供 选择的输出电压, 分别为 $2.35,3.3,4.1$ 和 $5 \mathrm{~V}$. 本文 选择 $2.35 \mathrm{~V}$ 的输出电压. 图 5 为升压转换电路的电路 图和实物图.

实验中, 使热电发生器冷端保持 $30^{\circ} \mathrm{C}$, 热端持续 升温至 $190^{\circ} \mathrm{C}$, 逐渐达到稳态后, 可输出 $100 \sim 110 \mathrm{mV}$ 的电量. 将热电发生器的输出端接到升压芯片输入 端后，发现升压芯片输出电压可达到 $2.38 \mathrm{~V}$, 足以驱 动 LED 灯 (见图 5(b)).

在实验中, 我们也注意到, 当热电发生器的输出 电压(即升压芯片输入电压)达到稳态后一段时间, 又 开始逐渐降低, 如图 6 所示. 这是由于组成热电偶的 导体上存在温度梯度，就不可避免地会发生不可逆 的热传导现象，从而导致当热电偶两端温差增大到 一定程度开始减小，进一步使得输出的热电势减小. 但从图 6 同时可以看出, 在 $67 \mathrm{~min}$ 的较长时间内, 虽 然热电发生器的输出电压从 $41 \mathrm{mV}$ 逐渐降落到 $38 \mathrm{mV}$, 

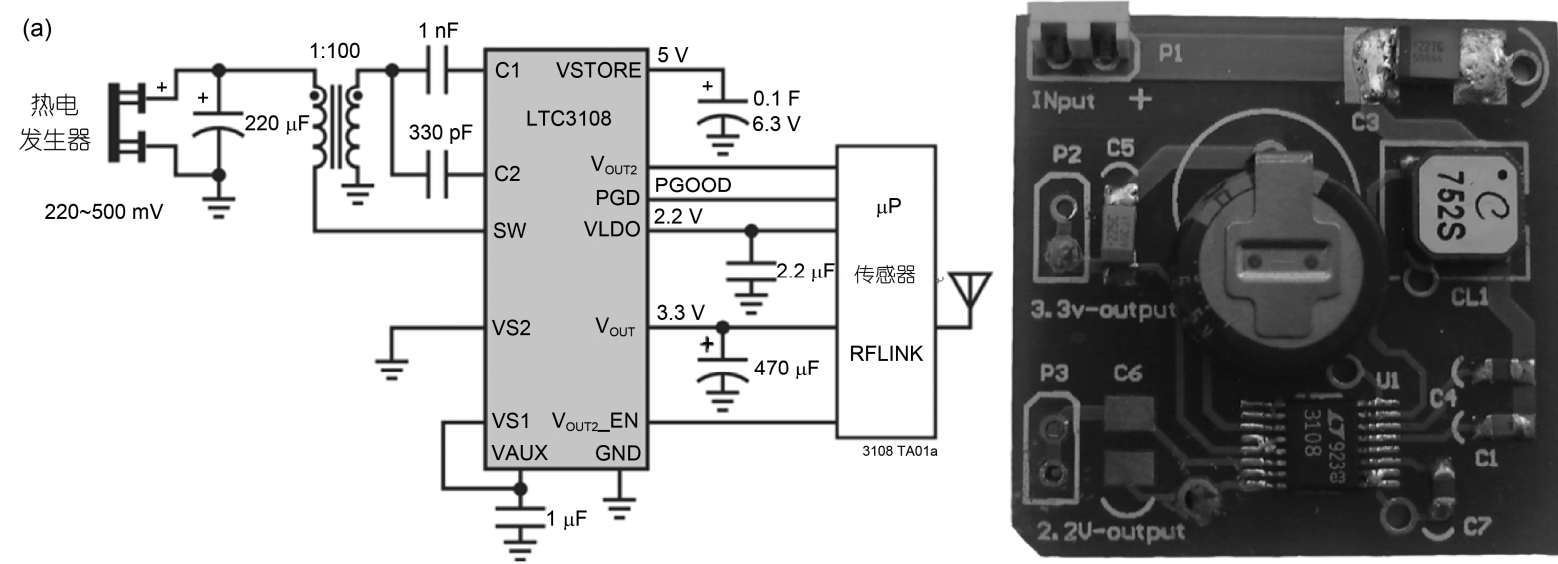

(b)

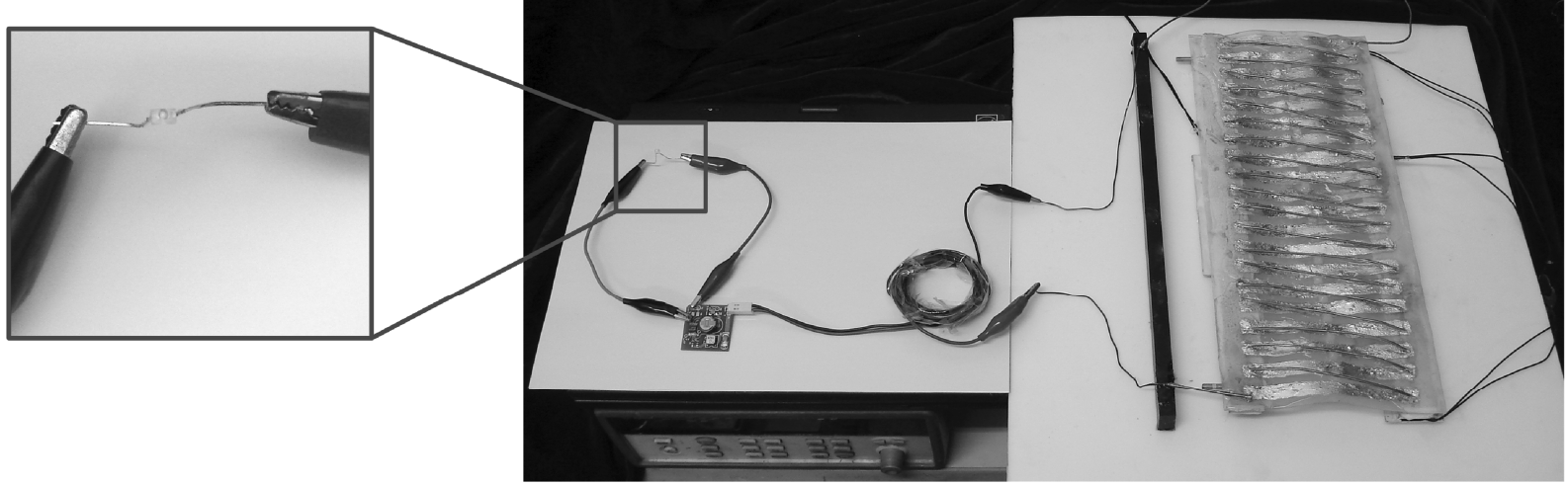

图 5 直写式热电发生器

(a) 升压型 $\mathrm{DC} / \mathrm{DC}$ 转换器; (b) 镓-康铜热电发生器改进原型机

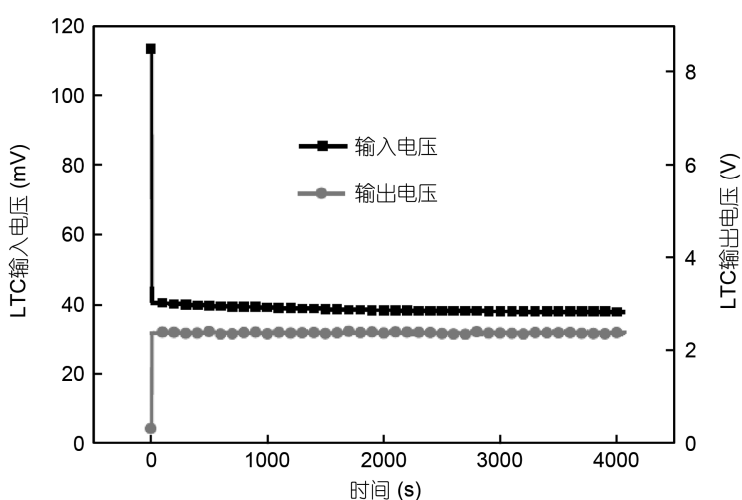

图 6 热电发生器升压芯片输入及输出电压随时间变化情况

但升压后的输出电压稳定在 $2.38 \mathrm{~V}$ 左右, 可以保证 LED 灯维持正常工作, 可见本文开发的热电发生器 是可靠的.

至此, 我们已经证明, 直写式热电发生器能够可 靠地驱动 LED 灯. 为进一步得到热电发生器为 LED
灯提供的功率, 我们将一个 $10 \Omega$ 的取样电阻和 LED 灯串联, 当升压电路输入电压为 $117 \mathrm{mV}$ 时, 测得 LED 灯和取样电阻端电压分别为 $1.59 \mathrm{~V}$ 和 $443 \mu \mathrm{V}$. 则 LED 灯的功率为

$$
w=E_{\mathrm{LED}} \cdot \frac{E_{q}}{R_{q}}=1.59 \mathrm{~V} \times \frac{443 \times 10^{-6} \mathrm{~V}}{10 \Omega}=70.44 \mu \mathrm{W},
$$

其中 $E_{L}$ 和 $E_{\mathrm{LED}}$ 分别为取样电阻和 LED 灯上的电压, $R_{L}$ 为取样电阻阻值. 可见, 该热电发生器可稳定供给 LED 灯 $70.44 \mu \mathrm{W}$ 电量, 足以保证其正常工作.

热电发生器达到稳态后的能量转换效率可计算为

$$
\begin{aligned}
\eta & =\left(1-T_{l} / T_{h}\right)\left[\frac{\sqrt{1+Z \bar{T}}-1}{\sqrt{1+Z \bar{T}}+T_{l} / T_{h}}\right]=(1-303 / 463) \\
& \times\left[\frac{\sqrt{1+3.82 \times 10^{-5} \times(303+463) / 2}-1}{\sqrt{1+3.82 \times 10^{-5} \times(303+463) / 2}+303 / 463}\right]=0.15 \% .
\end{aligned}
$$


可见, 虽然这里研制的热电发生器可以提供实 际应用所需的电压和功率, 但由于镓-康铜热电偶的 热电优值较低, 导致整个装置的转换效率偏低. 为提 高转换效率, 有必要进一步提高热电优值.

\section{3 液态金属直写式热电发生器性能的改进方案}

以上采用直写方法研制的热电发生器, 其薄膜 厚度一般为 $10 \mu \mathrm{m}$ 左右. 而膜厚通常直接影响到热电 偶的电阻, 从而决定了回路电流的高低, 继而限制了 可输出功率的大小. 为澄清此问题, 如下考察了镓薄 膜厚度对输出功率的影响.

这里热电发生器制作步骤如下: 1) 首先用 $0.8 \mathrm{~mm}$ 厚的硅胶板根据所需形状制作掩膜, 并使用 704 胶将 其黏接在 $3 \mathrm{~mm}$ 厚的硅胶基底上, 形成 20 个深度为 $0.8 \mathrm{~mm}$ 的槽道; 2) 用笔刷将液态镓涂覆填充槽道, 即镓膜层厚度为 $0.8 \mathrm{~mm} ; 3)$ 将康铜丝搭接在每两个 充满镓的槽道之间, 保证康铜丝两端与液态镓两端 相接；4）最后，使用 705 硅橡胶封装镓部分，完成装 置制作.

鉴于镓薄膜厚度相比上节的原型机已增大 80 倍, 为保证掩膜结构的稳定性, 将镓膜宽度从 $10 \mathrm{~mm}$ 减 至 $5 \mathrm{~mm}$, 而将两镓膜间距离从 $5 \mathrm{~mm}$ 增至 $10 \mathrm{~mm}$. 这 时镓热电极尺寸为 $100 \mathrm{~mm} \times 5 \mathrm{~mm} \times 800 \mu \mathrm{m}$, 康铜热 电极尺寸 $100 \mathrm{~mm} \times \Phi 1.5 \mathrm{~mm}$.

图 7 所示为由此改进后的热电发生器的热电势温差关系曲线. 从图 7 中可以看到, 当温差为 $140^{\circ} \mathrm{C}$ 时, 20 对同样尺寸的热电偶串联可得到输出电压约为 $123 \mathrm{~V}$, 已远高于 20 对镓热电极尺寸为 $100 \mathrm{~mm} \times$ $10 \mathrm{~mm} \times 10 \mu \mathrm{m}$ 的热电偶的输出电压的叠加, 从而为

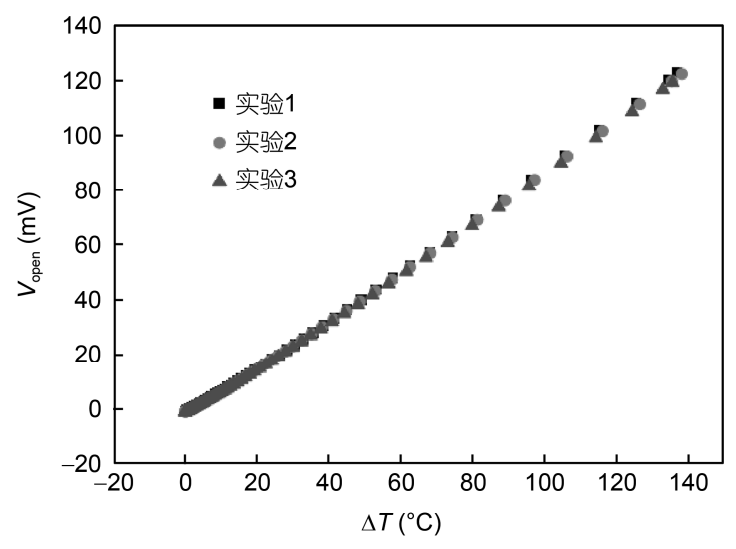

图 7 热电发生器热电势-温差关系曲线
驱动功能器件提供了更为可靠的保障.

仍然采用 LED 灯作为负载, 实验中, 使热电发 生器冷端保持 $30^{\circ} \mathrm{C}$, 热端持续升温至 $190^{\circ} \mathrm{C}$, 逐渐达 到稳态后, 可输出 110 120 mV 的电量, 将热电发生 器输出端接到升压芯片输入端后, 发现升压芯片输 出电压可达到 $2.38 \mathrm{~V}$, 可以驱动 LED 灯, 如图 8 所示.

与前述情况类似, 当热电发生器的输出电压(即 升压芯片输入电压)达到稳态后一段时间, 又开始降低. 但同时也可以看出, 在 $26 \mathrm{~min}$ 的较长时间内, 虽然热 电发生器的输出电压从 $111 \mathrm{mV}$ 逐渐降落到 $95 \mathrm{mV}$, 但升压后的输出电压稳定在 $2.38 \mathrm{~V}$, 可以保证 LED 灯稳定正常工作, 可见热电发生器是可靠的. 实际应 用过程中, 此时间段后的热电发生器驱动电压可达 到稳定工作状态. 当然, 若为确保自起始开始的任意 时段该发生器均能输出同一大小的电压, 还可通过 在输出电路上引入稳压芯片来实现, 具体细节此处 不再赘述.

另外, 当升压电路输入电压为 $115 \mathrm{mV}$ 时, 测得 LED 灯和 $10 \Omega$ 取样电阻端电压分别为 $1.70 \mathrm{~V}$ 和 $4.37 \mathrm{mV}$, 比 4.2 节开发的热电发生器分别提升 $6.92 \%$ 和 $886 \%$. 这时 LED 灯的功率为

$$
w=E_{\mathrm{LED}} \cdot \frac{E_{q}}{R_{q}}=1.70 \mathrm{~V} \times \frac{4.37 \times 10^{-3} \mathrm{~V}}{10 \Omega}=742.9 \mu \mathrm{W} .
$$
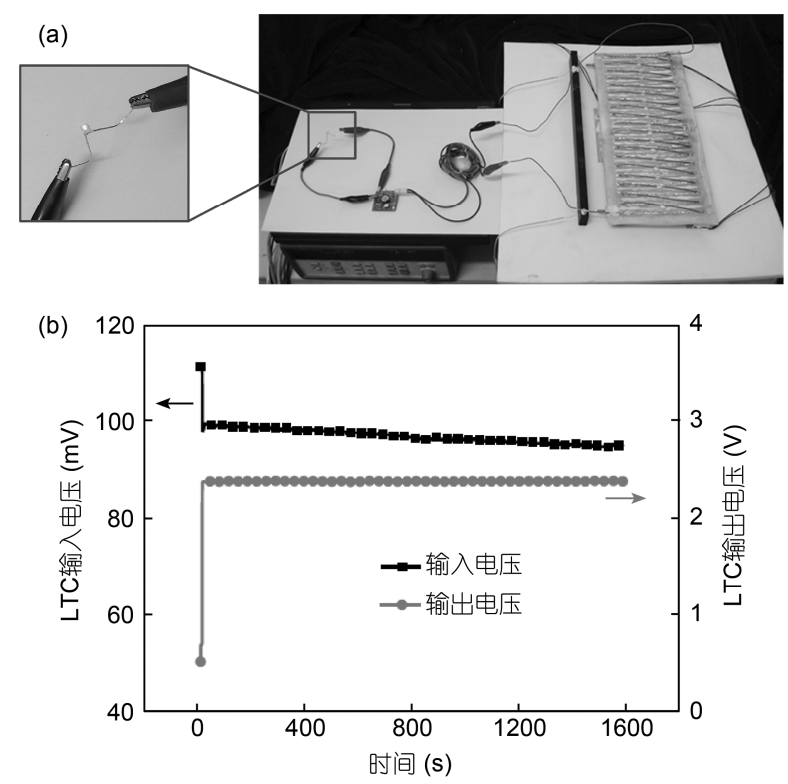

图 8 热电发生器工作情况

(a) 给 LED 灯供电; (b) 热电发生器升压芯片输入及输出电压随时 间变化情况 
可见, 性能提升后的热电发生器可稳定供给 LED 灯 $742.9 \mu \mathrm{W}$ 的电量, 比之前提升 $955 \%$, 说明提 高镓膜层厚度对输出功率的影响较大, 可作为一种 改进热电发生器的方法. 比较图 5 和 8 可发现, 本节 开发的热电发生器驱动的 LED 灯明显具有更高亮度, 亦证明了这一点.

另外, 通过观察液态镓-康铜热电偶以及镓-镓铟 合金热电偶的功率输入曲线, 我们发现输出功率与 温差的关系呈指数变化趋势. 温差小时发电功率很 小, 而随着温差的增大, 发电功率急速增加, 所以镓 基热电偶构成的热电发生器更适合于 $100^{\circ} \mathrm{C}$ 以上场 合的应用. 但由于受聚合物降解温度限制, 聚合物基 底印刷热电偶只限于 $200^{\circ} \mathrm{C}$ 以下低品位热量的回收 ${ }^{[11]}$. 更高的温度可考虑采用其他耐热柔性基底, 如玻璃 丝布等. 但对于类似玻璃丝布的多孔基底, 为防止基 底正反面发生电连接, 在印刷液态金属前, 还可预先 在基底两面各涂覆一层耐高温涂料. 热电极材料亦可 印刷在基底同一面或两面，以适应不同场合的需要.

\section{6 总结}

本文建立了可印刷式热电发生器技术, 并以镓康铜热电偶和镓-镓铟合金热电偶为代表, 系统探索 了利用直写方法制作的液态金属和固态金属所构成 的热电偶以及液态金属和液态金属合金之间构成的 热电偶的热量捕获性能, 在此基础上构建了镓-康铜 热电堆, 评估了热电堆的热量捕获性能.

通过串联 20 对镓膜厚度为 $10 \mu \mathrm{m}$ 左右的直写式 镓-康铜热电偶研制成热电发生器, 并对其输出电压 予以放大, 得到 $1.59 \mathrm{~V}$ 的负载电压和 $70.44 \mu \mathrm{W}$ 的功 率, 从而成功地驱动了 LED 灯, 证明了基于液态金 属热电偶的热电发生器用于捕获热量的可行性.

进一步地, 将镓膜层厚度从 $10 \mu \mathrm{m}$ 增大为 $800 \mu \mathrm{m}$, 成功地将负载电压和功率提升至 $1.70 \mathrm{~V}$ 和 $742.9 \mu \mathrm{W}$, 增率分别达 $6.92 \%$ 和 $955 \%$, 说明提高镓膜厚度可有 效提升输出功率, 这为今后进一步提升实用器件的 性能提供了一条有益途径.

\section{参考文献}

1 钱伯章. 新能源汽车与新型蓄能电池及热电转换技术. 北京: 科学出版社, 2010

2 Ono K, Suzuki R O. Thermoelectric power generation: Converting low-grade heat into electricity. J Miner Metals Mater Soc, 1998, 50: $49-51$

3 Yan H, Ohta T, Toshima N. Stretched polyaniline films doped by ( \pm )-10-camphorsulfonic acid: Anisotropy and improvement of thermoelectric properties. Macromolecular Materials Eng, 2001, 286: 139-142

4 Ismail B I, Ahmed W H. Thermoelectric power generation using waste-heat energy as an alternative green technology. Recent Patents Elec Eng, 2009, 2: 27-29

5 Jia D, Liu J. Human power-based energy harvesting strategies for mobile electronic device. Front Energy Power Eng China, 2009, 3: 27-46

6 Lu B, Gung V C. Online and remote motor energy monitoring and fault diagnostics using wireless sensor networks. IEEE Trans Indus Elec, 2009, 56: 4651-4659

7 Liu J C, Yao J. Wireless RF identification system based on SAW. IEEE Trans Indus Elec, 2008, 55: 958-961

8 Paradiso J A, Starner T. Energy scavenging for mobile and wireless electronics. IEEE Pervasive Comp, 2005, 4: 18-27

9 Watanabe S, Murakami A, Yamada S I. Development of eco-drive thermo. Micromechatronics, 2000, 44: 25-31

10 Yang Y, Wei X, Liu J. Suitability of a thermoelectric power generator for implantable medical electronic devices. J Phys D: Appl Phys, 2007, 40: 5790-5800

11 Nuwayhid R Y, Rowe D M, Min G. Low cost stove-top thermoelectric generator for regions with unreliable electricity supply. Renew Energ, 2003, 28: 205-222

12 Snyder G J. Small thermoelectric generators. Elec Soc Interface, 2008, 3: 54-56

13 Glatz W, Muntwyler S, Hierold C. Optimization and fabrication of thick flexible polymer based micro thermoelectric generator. Sens Act A: Phys, 2006, 132: 337-345

14 Yadav A, Pipe K P, Shtein M. Fiber-based flexible thermoelectric power generator. J Power Sources, 2008, 175: 909-913

15 van Osch T H J, Perelaer J, de Laat A W M, et al. Inkjet printing of narrow conductive tracks on untreated polymeric substrates. Adv Materials, 2008, 20: 343-345

16 Rogers J A, Bao Z, Makhija A, et al. Printing process suitable for reel-to-reel production of high-performance organic transistors and 
circuits. Adv Materials, 1999, 11: 741-745

17 陈东勇, 应鹏展, 崔教林, 等. 热电材料的研究现状及应用. 材料导报, 2008, 22: 280-282

18 Markowski P, Dziedzic A. Planar and three-dimensional thick-film thermoelectric microgenerators. Microelectron Reliab, 2008, 48: 890-896

19 Silva M F, Ribeiro J F, Carmo J P, et al. Thin Films for Thermoelectric Applications. In: Bhushan B, ed. Scanning Probe Microscopy in Nanoscience and Nanotechnology. Berlin Heidelberg: Springer-Verlag, 2013. 485-528

20 朱铁军, 赵新兵, 胡淑红. 新型热电材料 $\beta-\mathrm{Zn}_{4} \mathrm{Sb}_{3}$ 的电学性能. 稀有金属采用与工程, 2001, 30: 187-189

21 Majumdar A. Thermoelectricity in semiconductor nanostructures. Science, 2004, 303: 777-778

22 Vineis C J, Shakouri A, Majumdar A, et al. Nanostructured thermoelectrics: big efficiency gains from small features. Adv Mater, 2010, 22 : 3970-3980

23 Venkatasubramanian R, Siivola E, Colpitts T, et al. Thin-film thermoelectric devices with high room-temperature figures of merit. Nature, 2001, 413: 597-602

24 Markowski P, Dziedzic A, Prociów E. Mixed thick/thin film thermoelectric microgenerators. In: 2008 Electronics System-Integration Technology Conference. Greenwich, ESTC 2008. 601-606

25 Li H Y, Yang Y, Liu J. Printable tiny thermocouple by liquid metal gallium and its matching metal, Appl Phys Lett, 2012, 101: 073511

26 钱增源. 低熔点金属的热物性. 北京: 科学出版社, 1985

27 Ma K Q, Liu J. Liquid metal cooling in thermal management of computer chips. Front Energy Power Eng China, 2007, 1: 384-402

28 Horner P. Thermoelectric power of gallium. Nature, 1962, 193: 58-58

29 金齐 P A. 热电偶测温. 陈道龙, 译. 北京: 原子能出版社, 1980

30 Weber J, Potje-Kamloth K, Haase F, et al. Coin-size coiled-up polymer foil thermoelectric power generator for wearable electronics. Sens Act A: Phys, 2006, 132: 325-330

\title{
Liquid metal based printable thermoelectronic generator and its performance evaluation
}

\author{
LI Hai Yan ${ }^{1}$, ZHOU Yuan ${ }^{2} \&$ LIU Jing ${ }^{1,2}$ \\ ${ }^{1}$ Key Laboratory of Cryogenics, Technical Institute of Physics and Chemistry, Chinese Academy of Sciences, Beijing 100190, China; \\ ${ }^{2}$ Department of Biomedical Engineering, School of Medicine, Tsinghua University, Beijing 100084, China
}

As the newly emerging direct writing technology, the liquid metal printed electronics is showing large promising potential in quickly manufacturing a variety of flexible electronics and circuits, optoelectronic sensors as well as energy harvesting devices etc. Here, the concept of liquid metal enabled directly printable thermoelectric generator was proposed for the first time and an integrated prototype device was fabricated. Taking thermocouple and thermopile directly made of gallium-constantan or gallium-GaIn alloy as typical examples, the thermoelectric performances of these two kinds of devices made of liquid-solid or liquid-liquid components were systematically evaluated and appropriate matching materials were identified. Further, 20 gallium-constantan thermocouples were integrated together as a thermoelectric generator. Through magnifying its output electric voltage, this device could offer a load voltage of $1.59 \mathrm{~V}$ and a power of $70.44 \mu \mathrm{W}$ which is capable of driving a LED lamp. In addition, when the gallium film depth was improved from its original 10 to $800 \mu \mathrm{m}$, significantly improved load voltage of $1.70 \mathrm{~V}$ and power of $742.9 \mu \mathrm{W}$ were obtained with increasing ratio as $6.92 \%$ and $955 \%$, respectively. The present experiments demonstrated the practical values of the liquid metal based printed thermoelectric generator in harvesting thermal energy.

\section{thermoelectric generator, heat harvesting, printed electronics, liquid metal, direct writing technology}

doi: 10.1360/092013-1239 